\title{
A top-down view on DNA replication and recombination from 9,000 feet above sea level
}

\author{
Erik Johansson', Christian Speck ${ }^{2}$ and Andrei Chabes ${ }^{1,3 *}$
}

\begin{abstract}
A report of the Keystone Symposium 'DNA Replication and Recombination' held in Keystone, USA, 27 February to 4 March 2011.
\end{abstract}

The triennial Keystone Symposium on 'DNA Replication and Recombination' brought together researchers working on various aspects of genome duplication, recombination and repair. A clear trend of this exciting meeting was a further shift from 'ovals and arrows' models to a more mechanistic understanding of the processes underlying DNA replication and recombination. This shift has been made possible in part by a growing number of solved protein structures, often in complex with DNA, and elegant single-molecule approaches. Several of the presentations revealed an intimate link between DNA replication and DNA recombination, bringing these two fields closer together than ever. Here we report a few of the highlights of the meeting.

\section{Keeping DNA replication on track}

The MCM2-7 protein complex is the precursor of the replicative helicase that unwinds DNA in front of the active replication complex. During the G1 phase of the cell cycle, the helicase is loaded in an inactive form onto DNA. In $S$ phase, MCM2-7 is activated by the cyclindependent and Dbf4-dependent kinases to become a processive replicative helicase. This activation requires the assembly of a large number of replication factors, culminating in the formation of the Cdc45-GINSMCM2-7 (CMG) complex, which has strong helicase activity in vitro. Michael Botchan (University of California, Berkeley, USA) presented three-dimensional electron microscopy structures of the entire Drosophila melanogaster CMG complex. Interestingly, Cdc45 and GINS bridge a weak interface between $\mathrm{Mcm} 2$ and $\mathrm{Mcm} 5$,

*Correspondence: andrei.chabes@medchem.umu.se

'Department of Medical Biochemistry and Biophysics, Umeå University, SE 90187

Umeå, Sweden

Full list of author information is available at the end of the article potentially sealing a gap between the two subunits. Using mutational data and structural information, Botchan suggested that the leading strand of the DNA passes through the central channel of the CMG complex. Data presented by Johannes Walter (Harvard Medical School, Boston, USA) also suggest that CMG operates by steric exclusion, with only the leading strand passing through the central channel of the complex. Thus, the two labs agree that the model in which the double-stranded DNA passes through the central channel of the helicase and becomes separated by a 'plough share' is not feasible. Botchan's work further indicates that $\mathrm{Cdc} 45$ has a structure similar to the bacterial recombination protein RecJ, linking replication and recombination. Cdc45 might be able to bind single-stranded DNA, which suggests that the lagging strand is channeled through Cdc45, whereas the leading strand passes through the center of the MCM ring. For the future, a more detailed understanding of the paths of the leading and lagging DNA strands running through the CMG helicase complex towards the DNA polymerases is needed.

\section{Role of BRCA2 in DNA replication}

The breast cancer suppressor protein BRCA2 has an important role in double-strand break repair via homologous recombination. Stephen Kowalczykowski (University of California, Davis, USA) described for the first time the purification of this impressive 3,418-amino-acid protein and reported that it binds RAD51 (the human homolog of bacterial RecA) and promotes RAD51 assembly onto single-stranded DNA. The purification of BRCA2 will allow the study of the functions of the different domains in this large protein.

Katharina Schlacher (Memorial Sloan-Kettering Cancer Center, New York, USA), presented elegant singlemolecule DNA fiber analysis demonstrating that BRCA2 protects newly synthesized DNA from degradation by the nuclease MRE11 when replication forks are stalled by hydroxyurea. Mutational analysis of BRCA2 by Schlacher and her colleagues enabled the separation of functions required for homologous recombination and for fork protection. Thus, BRCA2 maintains genomic integrity both through homologous recombination and by the protection of nascent strands during DNA replication. 


\section{Protein fusions bypass essential functions}

In the yeast Saccharomyces cerevisiae, DNA polymerase $\varepsilon$ (Pol $\varepsilon$ ) synthesizes primarily the leading strand, while DNA polymerase $\delta$ ( $\mathrm{Pol} \delta$ ) synthesizes primarily the lagging strand and the genes encoding the catalytic subunits of these two polymerases are essential for life. However, it has previously been shown that deletion of the amino-terminal part of the Pol 2 protein containing the Pol $\varepsilon$ catalytic domain resulted in sick but viable yeast cells, while deletion of the carboxy-terminal part of Pol2 was lethal. Hiroyuki Araki (National Institute of Genetics, Shizuoka, Japan) explored the essential function of the carboxy-terminal part of Pol2. Identification of a series of protein-protein interactions suggested that the Pol2 and Dpb2 subunits of Pol $\varepsilon$ function as structural mediators in the pre-loading complex (pre-LC). The pre-LC consists of Dpb11, Sld2, GINS and Pol $\varepsilon$, and experiments revealed that within this complex the carboxy-terminal part of Pol2 binds to Sld2 while Dpb2 binds to GINS, and that together Pol2 and Dpb2 bring Sld2 and GINS to replication origins. In a reductionist approach, different proteins within the pre-LC were fused together, demonstrating that the Sld2-Dpb2 fusion bypasses the requirement for the carboxy-terminal part of Pol2. A yeast strain lacking the POL2 gene is reported to be sick but viable. On the basis of these data the essential role of Pol $\varepsilon$ is proposed to be as a hub for GINS and Sld2 during the formation of the pre-LC.

Another example of the use of protein fusions was presented by Peter Burgers (Washington University School of Medicine, St Louis, USA), who reported a novel Fe-S cluster at the carboxyl terminus of Pol3 (the catalytic subunit of yeast Pol $\delta$ ) that is important for subunit interaction. He also reported a new interaction between one of the cysteine-rich motifs at the carboxyl terminus of Pol3 and PCNA, a protein that acts as a processivity factor for Pol $\delta$. Mutation of this cysteine-rich motif abolishes processive synthesis by Pol $\delta$ and leads to lethality in vivo. Interestingly, while the carboxy-terminal domain of Pol3 is essential, presumably because of its interaction with PCNA and other Pol $\delta$ subunits, the amino-terminal part of Pol3 containing the catalytic domain could be replaced in vivo by a DNA polymerase from bacteriophage RB69. This fusion protein will be useful for exploring functions of different domains in Pol $\delta$.

\section{Origin recognition complex - an origin of disease}

The origin recognition complex (ORC) is bound to DNA at the origins of replication. During the G1 phase of the cell cycle, the ORC functions with the proteins Cdc6 and Cdt1 to load the MCM2-7 helicase onto DNA, resulting in the formation of pre-replication complexes (pre-RC). No mutations in human genes encoding ORC proteins have been reported up to now. So the report by Andrew
Jackson (MRC Human Genetics Unit, IGMM, Edinburgh, UK) that the MCM2-7 loading factors Orc1, Orc4, Orc6, Cdt1 and Cdc6 are involved in Meier-Gorlin syndrome, an autosomal recessive primordial dwarfism syndrome, came as something of a surprise. Mutations in these proteins have been identified, and link defects in proteins of the pre-RC to reduced cell division and to abnormal development. So far, it is unclear whether the defect in Meier-Gorlin syndrome is due to a reduced proliferation rate of the patients' cells, or if the pre-RC proteins have an additional function in regulating development. However, it is clear that pre-RC proteins are relevant to human health, which warrants further detailed characterization of the mutations.

Thomas Kunkel (National Institute of Environmental Health Sciences, Research Triangle Park, USA) presented evidence that replicative DNA polymerases from $S$. cerevisiae are not perfect in their discrimination against the incorporation of ribonucleotides into DNA. In vitro studies suggest that $\operatorname{Pol} \alpha$, Pol $\delta$ and Pol $\varepsilon$ collectively incorporate more than 10,000 ribonucleotides into the yeast genome during each round of replication. Kunkel described how mutation of the Pol $\varepsilon$ catalytic-site residue responsible for discrimination of ribonucleotides allowed the selection of two Pol $\varepsilon$ variants, one that discriminated ribonucleotides better and another that discriminated them less well than the wild-type polymerase. To investigate ribonucleotide incorporation in vivo, the genes for the two Pol $\varepsilon$ variants were integrated into the yeast genome and the stability of DNA from the two strains towards alkali treatment was analyzed. The data presented by Kunkel indicate that incorporation of ribonucleotides into the genome occurs during DNA replication in vivo and that ribonucleotides are removed by an RNaseH2-dependent mechanism.

In conclusion, this successful symposium brought a great amount of molecular detail into the pathways involved in the initiation and progression of DNA replication, DNA recombination, DNA repair and cell cycle. The dual role of BRCA2 in DNA recombination and DNA replication fork stability, and structural similarities between the proteins involved in DNA replication and DNA recombination are signs of a growing overlap between these two fields.

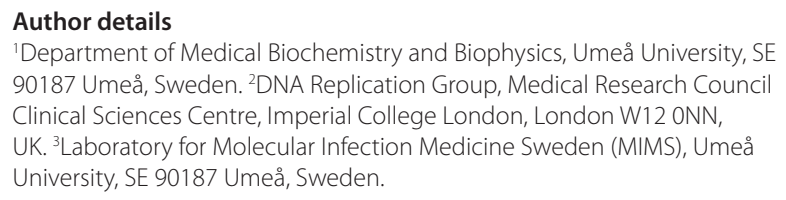

Published: 27 April 2011

doi:10.1186/gb-2010-12-4-304

Cite this article as: Johansson $\mathrm{E}$, et al:: A top-down view on DNA replication and recombination from 9,000 feet above sea level. Genome Biology 2011, 12:304. 